Honam Mathematical J. 36 (2014), No. 3, pp. 659-668

http://dx.doi.org/10.5831/HMJ.2014.36.3.659

\title{
THE GREEN FUNCTION AND THE SZEGÖ KERNEL FUNCTION
}

\author{
YounG-BOK CHUnG
}

\begin{abstract}
In this paper, we express the Green function in terms of the classical kernel functions in potential theory. In particular, we obtain a formula relating the Green function and the Szegö kernel function which consists of only the Szegö kernel function in a $C^{\infty}$ smoothly bounded finitely connected domain in the complex plane.
\end{abstract}

\section{Introduction}

It is a classical fact (see [5]) that the Green's function $g_{\Omega}(z, a)$ associated to a $C^{\infty}$ smoothly bounded finitely connected domain $\Omega$ and a singular point $a \in \Omega$ is related to the classical Bergman kernel function $K(z, a)$ associated to $\Omega$ via

$$
K(z, a)=\frac{-2}{\pi} \frac{\partial^{2} g_{\Omega}(z, a)}{\partial z \partial \bar{a}} .
$$

Since the Bergman kernel function also relates to the Szegö kernel function in an explicit way (see [5] again), the Green function can be essentially represented in terms of the Szegö kernel function. In fact, Bell [1] proved that the classical Drichlet problem can be solvable in terms of the Szegö kernel function and he found an explicit formula for the solution of the Dirichlet problem in terms of the Szegö projection which implies that the Green function is represented in terms of the Szegö projection.

In this paper, we find an explicit formula relating the Green function and the Szegö kernel function in more detail and in more readable way. In particular we would like to obtain an identity between them which

Received July 31, 2014. Accepted August 29, 2014.

2010 Mathematics Subject Classification. Primary 30C40.

Key words and phrases. Green function, Szegö kernel, Ahlfors map, Dirichlet problem. 
consists of only the Szegö kernel function in a $C^{\infty}$ smoothly bounded finitely connected domain.

\section{Preliminaries and Some Notations}

Suppose that $\Omega$ is a finitely connected bounded domain in the plane with $C^{\infty}$ smooth boundary. The Cauchy integral formula says that for any homomorphic function $f$ in a neighborhood of $\bar{\Omega}$ and for any point $a$ in $\Omega$, the value of $f$ at $a$ is represented by the boundary values of $f$ via

$$
f(a)=\frac{1}{2 \pi i} \int_{b \Omega} \frac{f(z)}{z-a} d z .
$$

If we introduce the classical $L^{2}$ inner product $<,>$ defined by

$$
<u, v>=\int_{b \Omega} u \bar{v} d s
$$

where $d s$ is the differential element of arc length on the boundary $b \Omega$, the integral formula (2.1) is equivalent to the identity

$$
f(a)=<f, C_{a}>,
$$

where $C_{a}(z)=\overline{\frac{1}{2 \pi i} \frac{T(z)}{z-a}}$ is the Cauchy kernel and $T$ is the unit tangent vector function on $b \Omega$ pointing in the direction of the standard orientation of $b \Omega$. This motivates to studying on the Hardy space of the boundary of $\Omega$ as follows.

Let $L^{2}(b \Omega)$ be the Hilbert space completion of $C^{\infty}(b \Omega)$ with respect to the inner product $\left\langle\cdot, \cdot>\right.$ and let $H^{2}(b \Omega)$ denote the classical Hardy space associated to $\Omega$ which is the space of holomorphic functions on $\Omega$ with $L^{2}$-boundary values in $b \Omega$. Since $H^{2}(b \Omega)$ is regarded as the completion of the restrictions of holomorphic functions in $C^{\infty}(\bar{\Omega})$ to $b \Omega$ in $L^{2}(b \Omega)$, it follows from the inequality $|f(a)| \leq\|f\|_{L^{2}(b \Omega)}\left\|C_{a}\right\|_{L^{2}(b \Omega)}$ that the evaluation function at $a \in \Omega$ is a continuous linear functional on $H^{2}(b \Omega)$. Thus, given $a \in \Omega$, we can apply the Riesz Representation Theorem to the linear functional on $H^{2}(b \Omega)$ to get a unique function $S_{a} \in H^{2}(b \Omega)$ such that for all $f \in H^{2}(b \Omega)$,

$$
f(a)=<f, S_{a}>=\int_{b \Omega} f \overline{S_{a}} d s .
$$

On the other hand, since $H^{2}(b \Omega)$ is a closed subspace of $L^{2}(b \Omega)$, there exists the orthogonal projection of $L^{2}(b \Omega)$ onto $H^{2}(b \Omega)$ called the Szegö 
projection which is denoted by

$$
P: L^{2}(b \Omega) \rightarrow H^{2}(b \Omega) .
$$

Since for all $f \in H^{2}(b \Omega)$,

$$
<f, S_{a}>=f(a)=<f, C_{a}>=<f, P\left(C_{a}\right)>
$$

and $P\left(C_{a}\right) \in H^{2}(b \Omega)$, the uniqueness property for the function $S_{a}$ implies that

$$
P\left(C_{a}\right)=S_{a}
$$

and we call $S_{a}$ the Szegö kernel for the the Szegö projection $P$ and is denoted by $S_{a}(z)=S(z, a)$.

It is well known (see [1], [2]) that any $u \in L^{2}(b \Omega)$ has an orthogonal decomposition as a direct sum of the Hardy space $H^{2}(b \Omega)$ and the orthogonal complement $H^{2}(b \Omega)^{\perp}$ of the Hardy space via

$$
u=P(u)+\bar{T} \overline{P(\overline{u T})} .
$$

There is also a special kernel function which is the kernel for the orthogonal projection $P^{\perp}$ of the Szegö projection $P$ in a sense. The Garabedian kernel function $L(z, a)$ is defined by

$$
L(z, a)=\frac{1}{2 \pi(z-a)}+P\left(\overline{i C_{a} T}\right)(z)=\frac{1}{2 \pi(z-a)}+<\overline{i C_{a} T}, S_{z}>.
$$

It thus follows that the Garabedian kernel function is written in terms of the single Szegő kernel function via

$$
\begin{aligned}
L(z, a) & =\frac{1}{2 \pi}\left(\frac{1}{z-a}-\int_{b \Omega} \frac{S(z, \zeta)}{\zeta-a} d s_{\zeta}\right) \\
& =\frac{1-(z-a) \int_{b \Omega} \frac{S(z, \zeta)}{\zeta-a} d s_{\zeta}}{2 \pi(z-a)} .
\end{aligned}
$$

On the other hand, it is easy to see from (2.3) that for fixed $a \in \Omega, L(z, a)$ is a meromorphic function on $\Omega$ with a single simple pole at $z=a$ having residue $\frac{1}{2 \pi}$ which extends $C^{\infty}$ smoothly up to the boundary of $\Omega$. It is also known (see [1]) that $L(z, a)$ never vanishes for all $(z, a) \in \bar{\Omega} \times \Omega$ with $z \neq a$. An important property about the Szegö kernel and the Garabedian kernel to which we often refer in this paper is

$$
L(z, a)=i \overline{S(z, a)} \overline{T(z)}, \quad(z, a) \in \mathrm{b} \Omega \times \Omega .
$$


It is very interesting to see that when $\Omega$ is simply connected, given $a \in \Omega$, the quotient map

$$
f_{a}(z)=\frac{S(z, a)}{L(z, a)}
$$

is the Riemann mapping function associated to the pair $(\Omega, a)$ which is a biholomorphic mapping of $\Omega$ onto the unit disc with $f_{a}(a)=0$ and $f_{a}^{\prime}(a)>0$, having an extremal property which maximizes $h^{\prime}(a)$ among all holomorphic functions $h$ mapping $\Omega$ into the unit disc making $h^{\prime}(a)$ real valued (see $[6]$ ).

Now we want to study on the Green function for the purpose of expressing it in terms of the Sezgö kernel function. Let $g_{\Omega}(z, a)$ be the Green function associated to $(\Omega, a)$. It is harmonic in $\Omega \backslash\{a\}$, extends $C^{\infty}$ smoothly to the boundary of $\Omega$ and vanishes on $b \Omega$ such that $g_{\Omega}(z, a)+\ln |z-a|$ has a removable singularity at $a$. In virtue of the uniqueness of solution of the Dirichlet problem, it is easy to see that it is a conformally invariant quantity. In other words, if $\varphi: \Omega_{1} \rightarrow \Omega_{2}$ is a biholomophic mapping between $C^{\infty}$ bounded domains $\Omega_{1}$ and $\Omega_{2}$ in the plane, then

$$
g_{\Omega_{1}}(z, a)=g_{\Omega_{2}}(\varphi(z), \varphi(a)) .
$$

Notice that when $U$ is the unit disc, the Green function $g_{U}(z, 0)$ is obviously equal to $-\ln |z|$ and the Riemann mapping function $f_{(U, a)}(z)$ is equal to $\frac{z-a}{1-\bar{a} z}$. So, the Green function $g_{U}(z, a)$ associated to $(U, a)$ is given by

$$
g_{U}(z, a)=g_{U}\left(f_{(U, a)}(z), f_{(U, a)}(a)\right)=-\ln \left|\frac{z-a}{1-\bar{a} z}\right| .
$$

Hence in the case of a simply connected domain $\Omega$, the Green function associated to $(\Omega, \zeta)$ is given by

$$
g_{\Omega}(z, \zeta)=g_{U}\left(f_{(\Omega, a)}(z), f_{(\Omega, a)}(\zeta)\right)=-\ln \left|\frac{f_{(\Omega, a)}(z)-f_{(\Omega, a)}(\zeta)}{1-\overline{f_{(\Omega, a)}(\zeta)} f_{(\Omega, a)}(z)}\right| .
$$

In particular, letting $\zeta=a$ in the above identity and using $f_{(\Omega, a)}(z)=$ $\frac{S(z, a)}{L(z, a)}$ and (2.4), we can express the Green function associated to the simply connected domain $\Omega$ and the point $a \in \Omega$ in terms of only one 
function of the Sezgö kernel function via

$$
g_{\Omega}(z, a)=-\ln \left|f_{(\Omega, a)}(z)\right|=-\ln \left|\frac{2 \pi(z-a) S(z, a)}{1-(z-a) \int_{b \Omega} \frac{S(z, \zeta)}{\zeta-a} d s_{\zeta}}\right| .
$$

It is natural to ask whether we can do the same thing for the case of a finitely connected domain. There is a kind of generalization of the Riemann mapping function to a finitely connected domain which is called the Ahlfors map. For a finitely connected $n$-connected domain $\Omega$ in the plane with $C^{\infty}$ smooth boundary and $a \in \Omega$, the Ahlfors map $f_{a}$ (we use the same notation as the Riemann mapping function for convenience) associated to the pair $(\Omega, a)$ is the unique solution to the extremal problem: among all holomorphic functions $h$ mapping $\Omega$ into the unit disc, find the one making $h^{\prime}(a)$ real-valued and as large as possible. It is well known (see [6], [3], [4]) that the function $f_{a}$ is an $n$-to one proper holomorphic covering map of $\Omega$ onto the unit disc and is equal to the quotient

$$
f_{a}(z)=\frac{S(z, a)}{L(z, a)}
$$

of the Szeő kernel and Garabedian kernel functions.

\section{Main results}

Now suppose that $\Omega$ is a finitely connected $n$-connected domain in the plane, $n>1$ with $C^{\infty}$ smooth boundary and fix $a \in \Omega$. Note that the Ahlfors map $f_{a}$ has $n$ zeros in $\bar{\Omega}$ and the Garabedian kernel function $L(z, a)$ has a single simple pole at $z=a$ which does not vanish in $\bar{\Omega}$. It thus follows from (2.6) that $S(z, a)$ has $n-1$ zeroes in $\bar{\Omega}$ counting multiplicities. Let $a_{1}, a_{2}, \cdots, a_{k}$ be distinct zeroes of $S_{a}$ in $\Omega$ with multiplicities $m_{1}, m_{2}, \cdots, m_{k}$, respectively, such that $m_{1}+m_{2}+$ $\cdots+m_{k}=n-1$. Choose points $\beta_{1}, \beta_{2}, \cdots, \beta_{n-1}$ from each of the bounded connected components of the complement of $\bar{\Omega}$ in $\mathbb{C}$. For each $j=1,2, \cdots, n-1$, it follows from the orthogonal decomposition (2.2) of the function $S_{a} \ln \left|\cdot-\beta_{j}\right|$ that

$$
S_{a}(z) \ln \left|z-\beta_{j}\right|=P\left(S_{a} \ln \left|\cdot-\beta_{j}\right|\right)(z)+\overline{T(z)} \overline{P\left(\bar{T} \overline{S_{a}} \ln \left|\cdot-\beta_{j}\right|\right)(z)} .
$$


Then using the identity (2.5), we get

$\ln \left|z-\beta_{j}\right|=\frac{P\left(S_{a} \ln \left|\cdot-\beta_{j}\right|\right)(z)}{S_{a}(z)}+\overline{\left(\frac{P\left(\bar{T} \overline{S_{a}} \ln \left|\cdot-\beta_{j}\right|\right)(z)}{L_{a}(z)}\right)}, \quad z \in \mathrm{b} \Omega$.

Notice that the function $\ln \left|z-\beta_{j}\right|$ is harmonic in a neighborhood of $\bar{\Omega}$ and the second term of the right hand side of the above identity is antiholomorphic in $\Omega$ and its conjugate is in $H^{2}(b \Omega)$ because of the single simple pole of $L(z, a)$ at $z=a$. Since the set $\Omega$ is $n$-connected with $n>1$, it follows from the argument principle that the numerator of the first term

$$
P\left(S_{a} \ln \left|\cdot-\beta_{j}\right|\right)
$$

of the right hand side must have a pole at one of the zeroes $a_{1}, a_{2}, \cdots, a_{k}$ of $S_{a}$. Similarly as considering a linear combination of functions $\ln \mid z-$ $\beta_{j} \mid, j=1,2, \cdots, n-1$, we can also show that a linear combination of the functions $P\left(S_{a} \ln \left|\cdot-\beta_{j}\right|\right)(z), j=1,2, \cdots, n-1$ must have a pole at one of the zeroes $a_{1}, a_{2}, \cdots, a_{k}$. This implies that the system of linear equations

$$
\left(\begin{array}{ccc}
P\left(S_{a} \ln \left|\cdot-\beta_{1}\right|\right)\left(a_{1}\right) & \ldots & P\left(S_{a} \ln \left|\cdot-\beta_{n-1}\right|\right)\left(a_{1}\right) \\
P\left(S_{a} \ln \left|\cdot-\beta_{1}\right|\right)^{\prime}\left(a_{1}\right) & \cdots & P\left(S_{a} \ln \left|\cdot-\beta_{n-1}\right|\right)^{\prime}\left(a_{1}\right) \\
\cdot & \cdot & \cdot \\
\cdot & \cdot & \cdot \\
P\left(S_{a} \ln \left|\cdot-\beta_{1}\right|\right)^{\left(m_{1}-1\right)}\left(a_{1}\right) & \cdots & P\left(S_{a} \ln \left|\cdot-\beta_{n-1}\right|\right)^{\left(m_{1}-1\right)}\left(a_{1}\right) \\
\cdot & \cdot & \cdot \\
\cdot & \cdot & \cdot \\
P\left(S_{a} \ln \left|\cdot-\beta_{1}\right|\right)\left(a_{k}\right) & \cdots & P\left(S_{a} \ln \left|\cdot-\beta_{n-1}\right|\right)\left(a_{k}\right) \\
P\left(S_{a} \ln \left|\cdot-\beta_{1}\right|\right)^{\prime}\left(a_{k}\right) & \cdots & P\left(S_{a} \ln \left|\cdot-\beta_{n-1}\right|\right)^{\prime}\left(a_{k}\right) \\
\cdot & \cdot & \cdot \\
\cdot & \cdot & \cdot \\
P\left(S_{a} \ln \left|\cdot-\beta_{1}\right|\right)^{\left(m_{k}-1\right)}\left(a_{k}\right) & \cdots & P\left(S_{a} \ln \left|\cdot-\beta_{n-1}\right|\right)^{\left(m_{k}-1\right)}\left(a_{k}\right)
\end{array}\right)\left(\begin{array}{c}
\lambda_{1} \\
\lambda_{2} \\
\cdot \\
\cdot \\
\cdot \\
\cdot \\
\cdot \\
\cdot \\
\cdot \\
\cdot \\
\cdot \\
\cdot \\
\cdot \\
\lambda_{n-1}
\end{array}\right)
$$

has a trivial solution $\lambda_{1}=\lambda_{2}=\cdots=\lambda_{n-1}=0$ and hence the coefficient matrix $\mathcal{A}$ of (3.1) is nonsingular.

Now let $\mu_{1}, \mu_{2}, \cdots, \mu_{n-1}$ be the solution of the system of equations 
$(3.2) \quad \mathcal{A}\left(\begin{array}{c}\mu_{1} \\ \mu_{2} \\ \cdot \\ \cdot \\ \cdot \\ \cdot \\ \cdot \\ \cdot \\ \cdot \\ \cdot \\ \cdot \\ \cdot \\ \cdot \\ \cdot \\ \mu_{n-1}\end{array}\right)=\left(\begin{array}{c}P\left(S_{a} \ln |\cdot-a|\right)\left(a_{1}\right) \\ P\left(S_{a} \ln |\cdot-a|\right)^{\prime}\left(a_{1}\right) \\ \cdot \\ \cdot \\ \cdot \\ P\left(S_{a} \ln |\cdot-a|\right)^{\left(m_{1}-1\right)}\left(a_{1}\right) \\ \cdot \\ \cdot \\ P\left(S_{a} \ln |\cdot-a|\right)\left(a_{k}\right) \\ P\left(S_{a} \ln |\cdot-a|\right)^{\prime}\left(a_{k}\right) \\ \cdot \\ \cdot \\ \cdot \\ P\left(S_{a} \ln |\cdot-a|\right)^{\left(m_{k}-1\right)}\left(a_{k}\right)\end{array}\right)$

Then for each $i=1, \cdots, k$,

$$
\begin{aligned}
& P\left(S_{a} \ln |\cdot-a|-\sum_{j=1}^{n-1} \mu_{j} S_{a} \ln \left|\cdot-\beta_{j}\right|\right)\left(a_{i}\right) \\
& =P\left(S_{a} \ln |\cdot-a|\right)\left(a_{i}\right)-\sum_{j=1}^{n-1} \mu_{j} P\left(S_{a} \ln \left|\cdot-\beta_{j}\right|\right)\left(a_{i}\right) \\
& =0
\end{aligned}
$$

and similarly for $i=1, \cdots, k$ and for $l=1, \cdots, m_{i}$,

$$
\left.\frac{d^{m_{i}-l}}{d z^{m_{i}-l}} P\left(S_{a} \ln |\cdot-a|-\sum_{j=1}^{n-1} \mu_{j} S_{a} \ln \left|\cdot-\beta_{j}\right|\right)\right|_{z=a_{i}}=0 .
$$

It then follows that the function

$$
\frac{P\left(S_{a} \ln |\cdot-a|-\sum_{j=1}^{n-1} \mu_{j} S_{a} \ln \left|\cdot-\beta_{j}\right|\right)}{S_{a}}
$$

is holomorphic in $H^{2}(b \Omega)$. We apply the orthogonal decomposition (2.2) to the function $S_{a} \ln |\cdot-a|-\sum_{j=1}^{n-1} \mu_{j} S_{a} \ln \left|\cdot-\beta_{j}\right|$ and divide by $S_{a}$ to obtain 


$$
\begin{aligned}
\ln |z-a| & -\sum_{j=1}^{n-1} \mu_{j} \ln \left|z-\beta_{j}\right| \\
= & \frac{P\left(S_{a} \ln |\cdot-a|-\sum_{j=1}^{n-1} \mu_{j} S_{a} \ln \left|\cdot-\beta_{j}\right|\right)(z)}{S(z, a)} \\
& +\frac{\frac{T(z)}{S(z, a)}}{P\left(\bar{T} \overline{S_{a} \ln |\cdot-a|-\sum_{j=1}^{n-1} \mu_{j} S_{a} \ln \left|\cdot-\beta_{j}\right|}\right)(z)}, z \in \mathrm{b} \Omega \\
= & \frac{1}{S(z, a)}, \frac{P\left(S_{a} \ln |\cdot-a|-\sum_{j=1}^{n-1} \mu_{j} S_{a} \ln \left|\cdot-\beta_{j}\right|\right)(z)}{\left(\frac{P\left(i \bar{T} \overline{S_{a} \ln |\cdot-a|-\sum_{j=1}^{n-1} \mu_{j} S_{a} \ln \left|\cdot-\beta_{j}\right|}\right)(z)}{L(z, a)}\right)},
\end{aligned}
$$

where we used the identity (2.5). Hence the holomorphic property of the first term of the above identity implies that the harmonic extension of the function $\ln |z-a|$ to $\bar{\Omega}$ is given by

$$
\begin{aligned}
\sum_{j=1}^{n-1} \mu_{j} \ln \mid z & -\beta_{j} \mid+\frac{1}{S(z, a)} P\left(S_{a} \ln |\cdot-a|-\sum_{j=1}^{n-1} \mu_{j} S_{a} \ln \left|\cdot-\beta_{j}\right|\right)(z) \\
+ & \frac{\left(\frac{P\left(i \bar{T} \overline{S_{a} \ln |\cdot-a|-\sum_{j=1}^{n-1} \mu_{j} S_{a} \ln \left|\cdot-\beta_{j}\right|}\right)(z)}{L(z, a)}\right) .}{} .
\end{aligned}
$$

Hence we have proved the following theorem.

Theorem 3.1. Let $n$ be a positive integer with $n>1$. Suppose that $\Omega$ is a finitely $n$-connected domain in the plane with $C^{\infty}$ smooth boundary. Let $a \in \Omega$ be fixed. Let $a_{1}, a_{2}, \cdots, a_{k}$ be zeroes of $S_{a}$ in $\Omega$ with multiplicities $m_{1}, m_{2}, \cdots, m_{k}$, respectively such that $m_{1}+m_{2}+$ 
$\cdots+m_{k}=n-1$. Let points $\beta_{1}, \beta_{2}, \cdots, \beta_{n-1}$ be chosen from each of the bounded connected components of the complement of $\bar{\Omega}$ in $\mathbb{C}$. by

Then the Green function $g_{\Omega}(z, a)$ associated to the pair $(\Omega, a)$ is given

$$
\begin{aligned}
& g_{\Omega}(z, a)=-\ln |z-a|+\sum_{j=1}^{n-1} \mu_{j} \ln \left|z-\beta_{j}\right| \\
& +\frac{1}{S(z, a)}\left\langle S(\cdot, a) \ln |\cdot-a|-\sum_{j=1}^{n-1} \mu_{j} S(\cdot, a) \ln \left|\cdot-\beta_{j}\right|, S(\cdot, z)\right\rangle_{b \Omega} \\
& +\frac{2 \pi(\bar{z}-\bar{a})\left\langle S(\cdot, z), i \bar{T} \overline{S_{a} \ln |\cdot-a|-\sum_{j=1}^{n-1} \mu_{j} S_{a} \ln \left|\cdot-\beta_{j}\right|}\right\rangle_{b \Omega}}{1+2 \pi(\bar{z}-\bar{a})\left\langle S(\cdot, z), i C_{a} T\right\rangle_{b \Omega}},
\end{aligned}
$$

where $\mu_{1}, \mu_{2}, \cdots, \mu_{n-1}$ are the constants satisfying

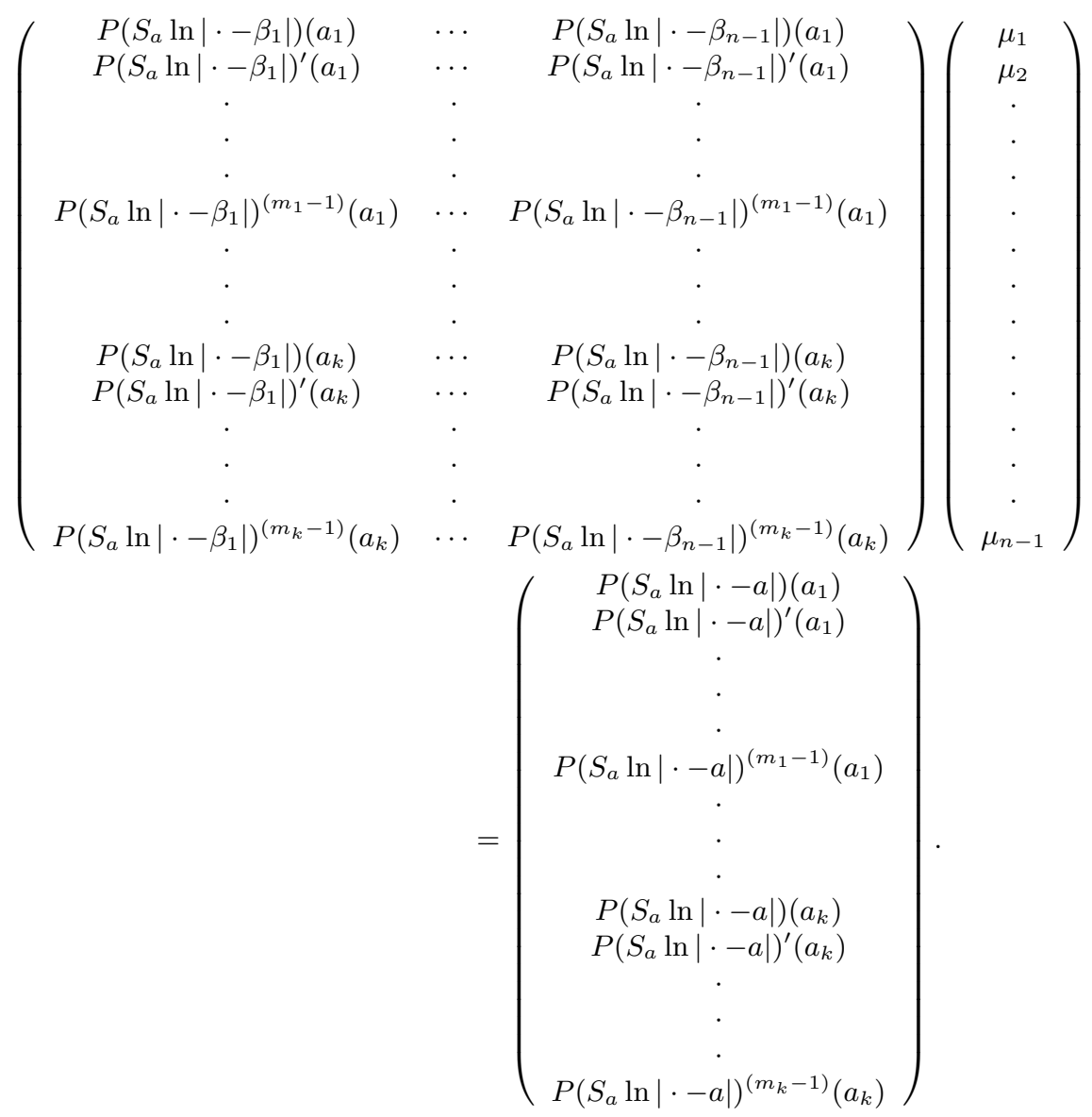




\section{References}

[1] S. Bell, Solving the Dirichlet problem in the plane by means of the Cauchy integral, Indiana Univ. Math. J. 39(4) (1990), 1355-1371.

[2] Steve Bell, The Szegö projection and the classical objects of potential theory in the plane, Duke Math. J. 64(1) (1991), 1-26. MR MR1131391 (93e:30018)

[3] - The Szegö projection and the classical objects of potential theory in the plane, Duke Math. J. 64(1) (1991), 1-26.

[4] Steven R. Bell, Ahlfors maps, the double of a domain, and complexity in potential theory and conformal mapping, J. Anal. Math. 78 (1999), 329-344. MR 1714417 (2000m:30012)

[5] Stefan Bergman, The Kernel Function and Conformal Mapping, Mathematical Surveys, No. 5, American Mathematical Society, New York, N. Y., 1950. MR $0038439(12,402 \mathrm{a})$

[6] P. R. Garabedian, Schwarz's lemma and the Szegö kernel function, Trans. Amer. Math. Soc. 67 (1949), 1-35.

Young-Bok Chung

Department of Mathematics, Chonnam National University,

Gwangju 500-757, Korea.

E-mail: ybchung@chonnam.ac.kr 